\section{RHEOLOGY OF RUBBER}

$\mathrm{R}$ UBBER is one of the many materials of great industrial importance the physical properties of which have not yet been precisely formulated, with the result that, although the word 'elastic' has become in everyday usage almost synonymous with 'rubber', the standard text-books on elasticity, if they refer to rubber at all, leave the impression that it is little more than a scientific curiosity.

Evidence that this state of affairs is rapidly passing was forthcoming from the large gathering of scientific men and technologists at a joint conference of the local section of the Institution of the Rubber Industry and the British Rheologists' Club, held in Manchester on April 17, to discuss some of the rheological problems of the rubber industry. At the morning session, at which Mr. W. N. Lister (I.R.I. local secretary) presided, papers were given by Dr. L. R. G. Treloar (British Rubber Producers' Research Association), Messrs. J. M. Buist and R. C. Seymour (Rubber Service Laboratories of I.C.I. (Dyestuffs) Ltd.) and Dr. J. R. Scott (Research Association of British Rubber Manufacturers).

In the first paper of the morning, Dr. Treloar dealt with "The Behaviour of Rubber in Elongation, Compression and Shear". His object was to show how these three simple types of deformation can be produced, and to consider the corresponding stress-strain relations, both from the experimental side and from the point of view of the molecular theory. His treatment referred only to well-vulcanized rubber, which can be regarded, at least approximately, as ideally elastic.

Dr. Treloar said that the fundamental reason for the high degree of elasticity of rubbers is to be found in the thermal motion of the chain atoms composing the very long molecules of which they are composed. This 'kinetic' theory shows that an assembly of molecules will take up a certain normal distribution of lengths which can be calculated by statistical methods, in much the same way as the molecules of a gas acquire a definite distribution of velocities. Any deformation of rubber disturbs this normal distribution, and hence leads to a less probable state. By considering an idealized network of cross-linked molecules Wall (J. Chem. Phys., 10, 485 ; 1942) has recently shown how the molecular distributions corresponding to elongation, uni-directional compression or shear may be described, and has calculated the probability of the distribution corresponding to any given state of deformation. By thermodynamic reasoning the probability has been related to the entropy, and this in turn to the work of deformation. The stress-strain relations are derived from the expression for the work of deformation.

The conclusions to be drawn. from the molecular network theory are that rubber should obey Hooke's law in shear, though not in elongation or compression. Wall showed also that the stress-strain relations for elongation and compression should form a single continuous curve. An interesting feature of Wall's formulæ is that the description of all three types of behaviour require the use of only one molecular constant, $M$, defined as the 'molecular weight' between junction points of the network. These conclusions are very illuminating, and must be regarded as of fundamental importance.

Dealing next with his experimental work, Dr. Treloar said that in comparing the experimental stress-strain relations with the theory it is desirable to use the same sample of rubber for the different types of deformation. He showed by practical demonstrations that a circular sheet of rubber clamped round its circumference can be inflated into a balloon, giving a 2-dimensional extension which is equivalent to a compression. A pure shear can be produced by applying a tension to a short wide strip of rubber gripped in wide clamps. The experimental stress-strain data for elongation and shear are found to be in agreement with Wall's formulæ when the deformations are not too large, but depart from them noticeably at higher deformations (for example, above 100 per cent elongation). For the compression the agreement is much closer. It is satisfactory to find that all three types of deformation lead to the same value of $M$.

Dr. Treloar believes that the experimental evidence shows that the basis of Wall's theory is substantially correct, but that modifications of it may be necessary to take account of certain properties of the molecules which the theoretical treatment does not at present include.

The second paper dealt with "The Position of the Rubber-like State on the Plastic-elastic scale". Mr. Seymour, who read the first part of this paper, began by referring to the classification of types of deformation put forward by the British Rheologists' Club (NATURE, 149, 702; 1942) which represents a type of scale in which ideal elasticity occurs as one extreme and Newtonian viscosity as the other. Rubbers occupy an intermediate position, being either viscoor plasto-elastic. But rubbers are not adequately described simply as being intermediate between solids and liquids, for whereas Hookean solids and Newtonian fluids can be described by single physical constants, the characteristics of rubber-like materials depend upon strain and upon rate of strain. Thus, representation of the behaviour of rubbers requires more complex functions relating the three variables stress, strain and time.

The deformation of rubber in compression can be described by an equation of the Nutting - Scott Blair type, relating the shear $\sigma$ with the shearing stress $S$ and time $t$, namely :

$$
\sigma=\frac{1}{\psi} S^{\beta} t^{k}
$$

For rubbers, $\beta$ is approximately $1 \cdot 0$. There are thus two constants for a given material, $\psi$ and $k$, which can be determined experimentally, and can be regarded as characteristic of a given rubber. A diagram can be drawn, in which $k$ is plotted horizontally and $\psi$ vertically, to represent the rheological properties of different rubbers.

For rubbers in extension an equation was proposed of the form

$$
\psi^{\prime}=S c^{-k^{\prime}} \sigma^{k^{-1}},
$$

which takes account of rate of strain $(\dot{\sigma})$.

The second part of the paper, read by Mr. Buist, included experimental data which show that the equations given above represent the behaviour of natural and synthetic rubbers. A study of a number of rubbers reveals that those having low values of $k$ are relatively difficult to mill. Natural rubber is the most easily milled, and has the highest $k$ value. Continued milling reduces $\psi$ (that is, the rubber becomes more plastic), and the spread of $k$ values for different rubbers is reduced, the lowering of $k$ for natural rubber being attributed to oxygen vulcanization.

Vulcanization moves all rubbers towards the 
elastic end of the scale, giving lower values of $k$. An 'index of reinforcement' was defined as the ratio of $k$ before and after compounding and vulcanization. For natural rubber this index is higher than for synthetics. This is reflected in the greater difficulty experienced in vulcanizing the synthetics. The movement toward the elastic end of the scale was demonstrated by analysis of the partial vulcanization (that is, 'scorching') of rubbers in terms of $\psi$ and $k$ values.

Values of $\psi^{\prime}$ and $k^{\prime}$ in the case of extension are characteristic for each rubber, but differ from $\psi$ and $k$ for the same materials.

The data of Hahn and Gazdik (India Rubber World, 51,$103 ; 1941)$ on the slow creep of rubbers in shear give values of $k$ and $\psi$ which are in accord with the performance of the engine mounting compounds described by these authors. For a given value of $\psi$ the smallest ereep is obtained with the lowest value of $k$.

Dr. Scott's paper, entitled 'Rheological Problems in the Rubber Industry", discussed a variety of problems in which the rheological properties of rubber are of great importance, and showed how the better understanding of these properties might assist in the control of technological processes.

Dr. Scott took a different line from the previous speakers, representing the properties by a plot of $d \sigma / d t$ (rate of shear) against $S$ (shearing stress). One can recognize specific types of flow curve, such as viscous, pseudo-viscous, plastic, etc., which can be related to the suitability of the material in any given circumstances. In the plasticization of rubber, the incorporation of filling ingredients requires first, good wetting properties, which means high fluidity under the action of small forces, and secondly, the breaking down of aggregates of filler particles, which necessitates high shearing stresses, and therefore high viscosity. These apparently opposite requirements can be satisfied by a certain type of flow curve.

The moulding of unvulcanized rubber is related to other rheological properties. The moulded article must retain its shape. This requires (1) the absence of elastic recovery, and (2) the presence of a yield value, so that the article will not deform under its own weight. The latter property can be assessed from the flow curve. Other exumples of rheological significance are the coating of fabrics with rubber, which requires ready penetration into the fabric, and the building up of tyres, etc., which requires good self-adhesion, and means the flowing of one rubber surface into the other. Vulcanization represents the suppression of flow, while reclaiming may be regarded as the recovery of the flow properties which have been suppressed by vulcanization. Methods of assessing dégree of vulcanization, for example, by so-called 'permanent set' tests, and the study of reclaiming thus come within the scope of rheology.

Dealing with methods of measuring the flow characteristics of rubber, Dr. Scott discussed the Williams parallel plate plastometer, extrusion methods, and the Mooney (shearing disk) plastometer, and the sort of information which can be obtained from them. None of these methods provides a uniform rate of shear throughout the specimen, which he considers essential for the fundamental study of rheological properties. Finally, he suggested that the study of the rheological behaviour of rubbers in relation to technological processes on one hand, and to their molecular structure on the other, opens up the possibility of producing rubbers having the particular properties which the technologist may require for any given purpose.

A visit was made in the afternoon to the Rubber Service Laboratories of I.C.I. (Dyestuffs) Ltd., at Blackley, by permission of the delegate directors. Members of both societies were welcomed on arrival by Dr. W. J. S. Naunton. The principal aspects of the handling and testing of natural and synthetic rubbers were illustrated in the laboratories, by a number of demonstrations which emphasized the broad rheological field covered by rubber-like materials.

\section{RECENT ADVANCES' IN ORGANIC CHEMICAL METHODS}

A $\mathrm{T}$ a meeting of the London and South Eastern $A$ Counties Section of the Institute of Chemistry on April 21, Dr. E. R. H. Jones, of the Imperia] College of Science and Technology, gave a lecture on "Recent Advances in Organic Chemical Methods". $\mathrm{He}$ said that spectacular advances which have characterized contemporary organic chemistry are largely to be attributed to vast improvements in the technique of the isolation, purification, examination and synthesis of organic compounds, and both new and improved physical and chemical methods have been extensively utilized. It is ever the aim of the organic chemist to employ methods involving the mildest possible conditions and the minimum quantity of material, a trend determined principally by his growing preoccupation with labile compounds of biological importance.

Notable advances made in distillation technique are attributable to the influence of the ever-growing petroleum industry. Molecular distillation is becoming increasingly popular and has rendered possible the isolation of vitamin $\mathrm{A}$ in a crystalline form. Chromatographic analysis has enormously simplified the purification of both naturally occurring and synthetic organic compounds and has provided a valuable new criterion of purity. On the chemical side a number of new reagents have been developed for the separation of particular classes of organic compounds.

The contribution of microanalysis towards recent achievements cannot be easily over-emphasized, and has led inevitably to the universal introduction of micro-methods. Absorption spectroscopy has proved of great service in the isolation and determination of the structure of vitamins and hormones, and although our knowledge of the relationship between structure and light absorption is still largely empirical, a sufficient fund of information is available to give the method a high diagnostic value.

Greater selectivity can now be obtained following the discovery of hydrogenation catalysts of the Raney nickel type and more specific chemical methods of reduction, for example, sodium in liquid ammonia, and calcium-ammonia $\left(\mathrm{Ca}\left(\mathrm{NH}_{3}\right)_{6}\right)$, are being continually developed. The Meewein-Ponndorf reaction is of unique value for the reduction of sensitive aldehydes and ketones, and Oppenauer's demonstration that this reaction can be reversed provides a useful means of oxidizing unsaturated secondary alcohols to ketones which renders un necessary the protection of unsaturated linkages. Wide popularity has been accorded to such highly specific oxidizing agents as lead tetra-acetate, osmium tetroxide and selenium dioxide. 